\title{
Enabling health application accessibility through interface modalities for elderly
}

\author{
Safira Alsana*, Fitri Trapsilawati, and Titis Wijayanto \\ Department of Mechanical and Industrial Engineering, Universitas Gadjah Mada, Jl. Grafika, No. 2, Yogyakarta, Indonesia
}

\begin{abstract}
Along with the development of technology, the launching of new devices often leaves the usability problems for the elderly behind. It happens not only for devices but also its application, i.e., entertainment applications, health applications, learning applications, etc. Most of the time, the elderly find it hard to use due to its usability problems or unfamiliar feelings. This research focused on empirically investigating a health application interface so that elderly could use it easily without obstacles and help the treatment independently. Three interface modalities were tested, namely, textual, pictorial, and typing interfaces. The result showed that the option-based interfaces (i.e., textual and pictorial) led to shorter performance time than input-based interface (i.e., typing) among the three interfaces. Also, the pictorial interface has the highest SUS score. All respondents chose the pictorial interface as their first choice, enabling health application accessibility through the appropriate interface modality for the elderly.
\end{abstract}

Keywords: Elderly, health application, interface, usability

\section{Introduction}

The journey of industrial revolution 4.0 within the past few years has largely affected technological media development in society. It is proven by the development of smartphone-based applications that are often found in various kinds of software apps. The generated applications' forms are very diverse, ranging from learning apps, entertainment apps, healthcare apps, etc.

One of the widely used applications is the healthcare application. Its purpose is to improve the efficiency in monitoring the health condition of patients, or users in daily basis. With many types of healthcare applications that have been developed, then appeared the health application which is more specifically associated with the diabetes disease. Theoretically, diabetes is a progressive chronic disease characterized by the body's inability to perform control of the metabolism of carbohydrates, fats, and proteins. In conclusion, the body's inability would lead to hyperglycaemia (levels of high blood glucose) [1].

Based on the World Health Organization (WHO) data, the number of patients with diabetes in Indonesia has reached $8.426 \times 10^{6}$ since 2000 . The score is expected to be further increased in the year 2030 and predicted to reach more than $21.257 \times 10^{6}$ patients [2]. From the data showed, it can be concluded that some people do not realize how dangerous the disease of diabetes for health.

In fact, during daily life, patients usually are not aware that they have contracted the disease of diabetes. Because of such unconscious condition, people with diabetes are often late to visit doctors and only consult their doctor when it is already in the chronic phase. As consequence, the health application began to be developed in order to support self-monitoring for diabetes patients. Therefore, health application is an effective solution for people who have had diabetes to monitor the levels of blood sugar without direct assistance from a doctor [3].

Today, various kinds of healthcare applications can be used to support health-related activities such as consultation, body state monitoring, purchasing of medicine, etc. using a smartphone [4]. Any application must be designed to fit its users' needs and user friendly. However, many available health applications have not carefully considered usability aspects, thus making confusion and difficulty for their users. Moreover, this confusion can be even worse for elderly users whose body functions and motoric responses have been decreased [5]. This infers that design investigation of health application is necessary [6]. Essential aspects in usability include ease of use, learnability, effectiveness, efficiency, satisfaction, and ease of access [7].

The most important part of an application is an easy interface for the user or (user interface). The user interface is a platform where users do most of the functions offered by an app. Essentially, health application must provide satisfaction through an effective interface during its use [8].

The only Indonesia health application for diabetes patient is Teman Diabetes app. It has been recently launched in 2018. The display of Teman Diabetes app is shown in Figure 1. Teman Diabetes app could monitor blood sugar, food data, exercise and remind users of their medication. This research aimed to further enhance

\footnotetext{
* Corresponding author: safiraalsana@mail.ugm.ac.id
} 
the interface of Teman Diabetes app in order to improve its effectiveness, efficiency, and satisfaction of user while using it. To do so, several interface modalities were investigated in this research.

\section{Literature review}

Theoretically, the developer has to understand the usability principle before implementing it on a system. In Usability Testing, Human interactions and tools are important parts that must be fulfilled in the system designing. Usability testing is a qualitative analysis that determines how easily the user uses the interface of an application [9]. The purpose of usability testing is to achieve effectiveness, efficiency, and satisfaction.

Effectiveness is related to how successful the user reaches the goal using an application or tool. Efficiency is related to how smooth the user is to achieve goals, time in doing it. Satisfaction is related to the user's acceptance of the application or tool. Usability testing is conducted to evaluate whether an application or tool is suitable for user requirements or not. A prior research was conducted related to usability testing of an android application interface based on usability criteria. The evaluation results indicated that the value of usability acceptance by users is above 3 (above the middle value) on a scale of 5 . Therefore, it can be concluded that the results have a positive effect on increasing the level of acceptability of android applications [10].

Similar research was also carried out in investigating the importance of usability of the user interface (UI) in the design of educational applications designed for children aged $4 \mathrm{yr}$ to $5 \mathrm{yr}$. The study provided UI design guidelines of design elements and principles for designers and developers in developing children's education applications [11]. Furthermore, another research was conducted to analyze and evaluate easy-touse and friendly applications for parents [12].

In a nutshell, this research investigated several different interface modalities to facilitate daily monitoring for the elderly who suffer from diabetes in Indonesia. Numerous research about diabetes application has been well documented in other countries. However, it is not the case in Indonesia, where research related to healthcare application, particularly diabetes, remains limited. Therefore, this research would further examine the interface modalities for diabetes application.

\section{Methods}

The respondents in this research are five elderly (1 male, 4 females) with the age range from $50 \mathrm{yr}$ old to $63 \mathrm{yr}$ old $(\mathrm{M}=56, \mathrm{SD}=2.64)$. The participation was voluntary.

This research only developed the interface section for food monitoring. There were two food monitoring interfaces developed in this study using Figma, including textual and pictorial interface. Figma was a web-based application to develop an interface prototype for this research. In textual interface, participants were provided with textual options of food database. In pictorial interface, the participants were provided with the pictorial options of food database.

The independent variable in this research was interface modality that includes texting, pictorial, and typing modalities. Typing interface is the main interface in the current version of Teman Diabetes app, meanwhile, the textual and pictorial interface is the prototype versions.

The participants were requested to record their daily food intake for breakfast, lunch, and dinner. Each participant recorded their food using the three methods in random order to cancel out the carry-over effect. Figure 1 to Figure 3 shows the display of the interfaces.

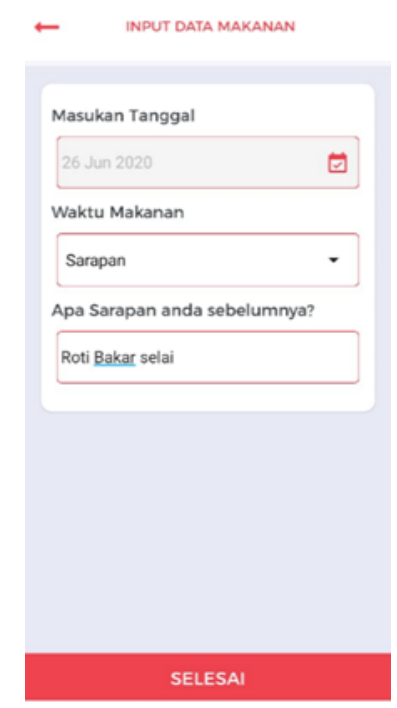

Fig. 1. Typing interface, the main interface of Teman Diabetes App

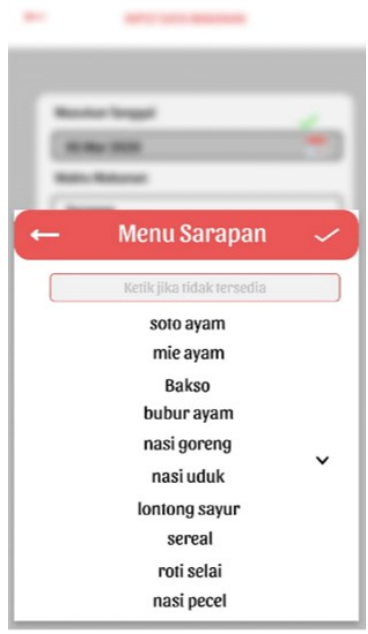

Fig. 2. Textual interface 


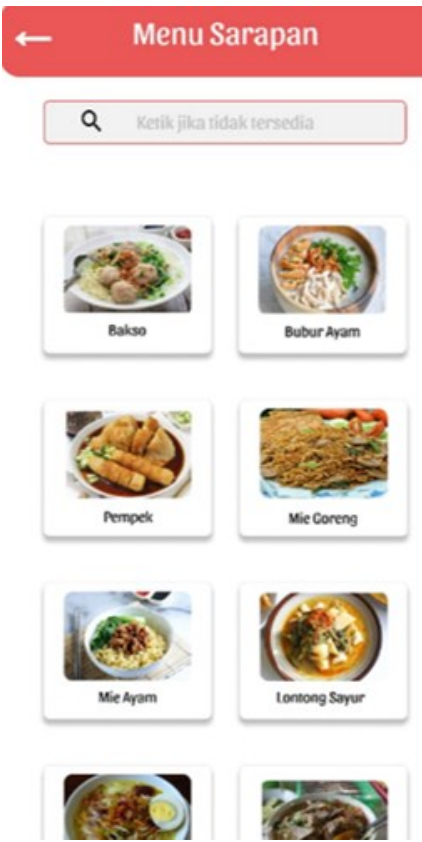

Fig. 3. Pictorial interface

\section{Results}

\subsection{Result of interface experiment}

\subsubsection{Result of textual interface}

Table 1. Success rate, duration and error of textual

\begin{tabular}{|c|c|c|c|}
\hline \multirow{2}{*}{$\begin{array}{c}\text { Respondent } \\
\text { List }\end{array}$} & \multicolumn{3}{|c|}{ Textual } \\
\cline { 2 - 4 } Respondent 1 & Success & Duration (s) & Error \\
\hline Respondent 2 & 1 & 35 & $0 \%$ \\
\hline Respondent 3 & 1 & 34 & $0 \%$ \\
\hline Respondent 4 & 1 & 30 & $0 \%$ \\
\hline Respondent 5 & 1 & 33 & $0 \%$ \\
\hline Average & & 33.8 & $0 \%$ \\
\hline
\end{tabular}

The success rate of the respondent in performing the task on the textual interface shown in Table 1. All respondents succeed in performing the task without error and the average duration was $33.8 \mathrm{~s}$.

\subsubsection{Result of pictorial interface}

Table 2. Success rate, duration and error of pictorial

\begin{tabular}{|c|c|c|c|}
\hline \multirow{2}{*}{$\begin{array}{c}\text { Respondent } \\
\text { List }\end{array}$} & \multicolumn{3}{|c|}{ Pictorial } \\
\cline { 2 - 4 } & Success & Duration (s) & Error \\
\hline Respondent 1 & 1 & 34 & $0 \%$ \\
\hline Respondent 2 & 1 & 36 & $0 \%$ \\
\hline Respondent 3 & 1 & 31 & $0 \%$ \\
\hline Respondent 4 & 1 & 36 & $0 \%$ \\
\hline Respondent 5 & 1 & 35 & $0 \%$ \\
\hline Average & & 34.4 & \\
\hline
\end{tabular}

The success rate of the respondent performing the task using the pictorial interface shown in Table 2. The average duration to complete the task was $34.4 \mathrm{~s}$. Its success rate was $100 \%$ with $0 \%$ of error.

\subsubsection{Result of typing interface}

Table 3. Success rate, Duration and Error of Typing

\begin{tabular}{|c|c|c|c|}
\hline \multirow{2}{*}{$\begin{array}{c}\text { Respondent } \\
\text { List }\end{array}$} & \multicolumn{3}{|c|}{ Typing } \\
\cline { 2 - 4 } Respondent 1 & Success & Duration (s) & Error \\
\hline Respondent 2 & 1 & 50 & $0 \%$ \\
\hline Respondent 3 & 1 & 49 & $0 \%$ \\
\hline Respondent 4 & 1 & 55 & $0 \%$ \\
\hline Respondent 5 & 1 & 47 & $0 \%$ \\
\hline Average & & 49.8 & $0 \%$ \\
\hline
\end{tabular}

All respondents succeed in performing the task on typing interface without error as shown in Table 3 . The average duration in completing the task was 49.9 s. It was the longest duration among all interfaces.

\subsection{Inferential analysis}

The inferential statistical test used was one-way ANOVA with a significance of $5 \%$. The results showed the significant effect of interface modality on the task duration, $\mathrm{F}(2.14)=59.6039, \mathrm{p}<.01$

A post-hoc test using Tukey HSD was conducted after the omnibus analysis. The post-hoc results indicated that typing interface required longer time duration significantly as compared to pictorial $(\mathrm{p}<.01)$ and texting $(p<.01)$. However, the durations required in textual and typing modalities were not different $(\mathrm{p}=.93)$.

\section{Discussions}

It is observed that all respondents have successfully performed the task using textual, pictorial and typing interfaces, respectively. The results showed that all interface modalities were effective in terms of its success rate.

\subsection{Duration of three interfaces}

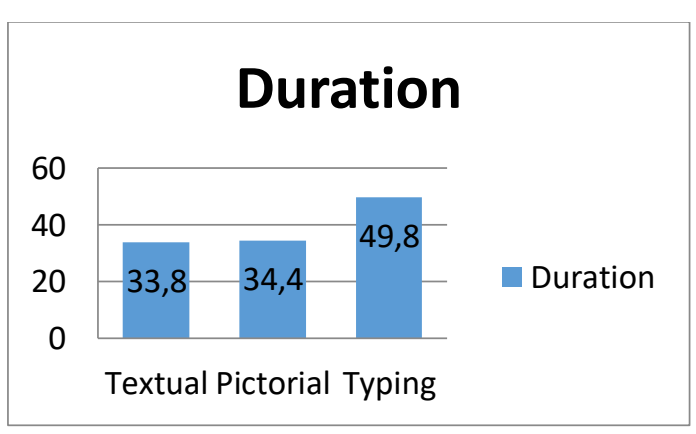

Fig. 4. Duration time 
For the task duration, all respondents could perform the task less than one minute. From Figure 4, it is shown that the participants could perform the task faster using textual and pictorial interfaces with $33.8 \mathrm{~s}$ and $34.4 \mathrm{~s}$, respectively. The task completion using typing interface required $49.8 \mathrm{~s}$.

This result inferred that the elderly performed the task efficiently with textual or pictorial. The gap between textual and pictorial is small. The Elderly completed the task the longest while using typing interface since they needed to type when inputting the menu, meanwhile textual and pictorial interface merely require tapping on appropriate menu.

These findings suggest that option-based interface was better than input-based interface for elderly users. This is also in line with the prior research [13] that compared clicking versus typing input. To examine which option-based interface is better, the further subjective analysis was done using SUS.

\subsection{System Usability Scale (SUS)}

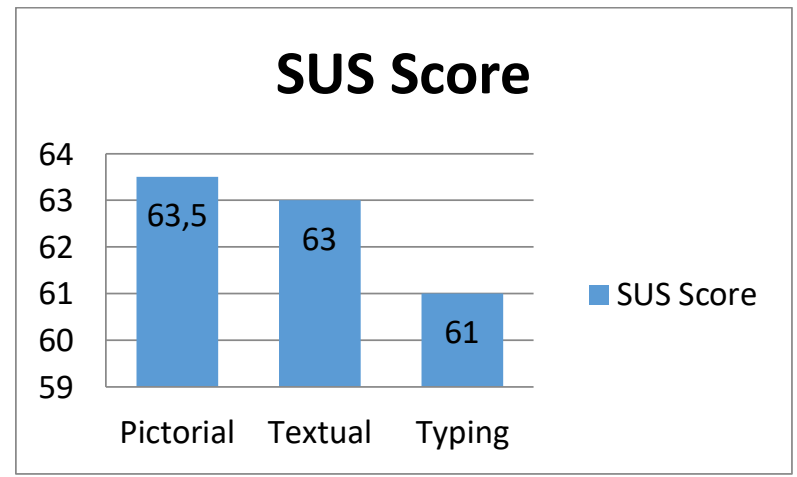

Fig. 5. System Usability Scale (SUS)

System Usability Scale (SUS) is a tool for assessing the usability (effectiveness, efficiency, and satisfaction) subjectively by respondents. Good category for SUS score is more than 70.4 [14]. The pictorial interface is at OK category with the score of 63.5. The pictorial interface has the highest SUS score as compared to other modalities. Although the difference was slight, the pictorial interface was also more preferred than textual interface. Further, the participants were also asked which interface they like most during the debriefing and most of them selected pictorial as their interface choice since it is more interesting and noticeable than textual and typing.

\section{Conclusion and future research}

This research aims to improve the usability aspect of Teman Diabetes for elderly population by creating a new design interface. Three interfaces tested by five respondents above $50 \mathrm{yr}$ old. Typing interface is the main interface of Teman Diabetes app. Pictorial and textual are the prototyping version of the interfaces. All respondents succeeded in performing the task with zero error. However, the typing interface required the longest time for task completion. The SUS' result showed that pictorial interface has the highest score. The participants also chose pictorial interface as the most attractive, easy to use and more noticeable than textual and typing interfaces.

This research has not examined other features of Teman Diabetes app such as blood sugar levels, exercise, medication reminder etc. It only investigated the interface in food data recording section. Therefore, future research will be done for the improvement of fullscale application.

\section{References}

1. J.S. Isaac, K. Porkumaran, Design Of Control Technique For Diabetes In Critical Care, International Conference on Green Computing Communication and Electrical Engineering (ICGCCEE), 1-4, (2014).

2. [Data Centres And Information Health Of The Republic Of Indonesia, World Diabetes Day, 2018. [Accessed Online on 20 May 2020]. Available:https://www.who.int/ehealth/about/en/.h ttps://pusdatin.kemkes.go.id/article/view/1904150 0002/hari-diabetes-sedunia-2018.html [Accessed on 20 March 2020].

3. K. Kalimullah, D. Sushmitha, Procedia Computer Science, 113, 352-359 (2017).

4. J.M. García-Gómez, I. Torre-Díez, J. Vicente, M. Robles, M. López-Coronado, J.J. Rodrigues, Health Informatics J., (2014).

5. J.C. Briede-Westermeyer, B. Pacheco-Blanco, M. Luzardo-Briceño, C. Pérez-Villalobos, Sustainability, 12, 7, 2690, (2020).

6. B. Maryem, E. Hakima, Y. Ikram, B. Mohamed, Diabetic patients and physicians' acceptability of a mobile health application for Diabetes monitoring in Fez region (Morocco), 1st International Conference on Innovative Research in Applied Science, Engineering and Technology (IRASET), (2020).

7. J. Rubin, D. Chisnell, Handbook of Usability Testing, Second Edition: How to Plan, Design, and Conduct Effective Test, Wiley Publishing Inc., Indianapolis, (2008).

8. J. Preece, Y. Rogers, H. Sharp, Interaction Design: Beyond Human Computer Interaction, John Wiley \& Sons Inc., New York, (2002).

9. J. Nielsen, Usability 101: Introduction to usability, 2012. [Accessed Online on 20 May 2020]. Available:

http://www.nngroup.com/articles/usability-101introduction-to-usability/

10. D.R. Rahadi, Journal of Information Systems (JSI), 6, 661-671 (2014).

11. M. Masooda, M. Thigambaramb, Procedia - Social and Behavioral Sciences, 197, 1818-1826 (2015).

12. J.M.D. Bossini, L. Moreno, Procedia Computer Science, 27, 57 - 66 (2014).

13. M.D. Hafiz, A.H. Abdullah, N. Ithnin, H.K. Mammi, Towards identifying usability and security features of graphical password in knowledge based 
authentication technique, In: 2008 Second Asia

International Conference on Modelling \&

Simulation (AMS). IEEE, pp. 396-403 (2008).

14. A. Bangor, K. Philip, J. Miller, Journal of usability studies (2009). 